\title{
The probabilistic relationship between the assignment and asymmetric traveling salesman problems
}

\author{
Alan Frieze* Gregory B. Sorkin ${ }^{\dagger}$
}

\section{Introduction}

The Assignment Problem (AP) is the problem of finding a minimum-weight perfect matching in an edge-weighted bipartite graph. An instance of the AP can be specified by an $n \times n$ matrix $C=(C(i, j))$; here $C(i, j)$ represents the weight (or "cost") of the edge between $i \in X$ and $j \in Y$, where $X, Y$ are disjoint copies of $[n]=\{1,2, \ldots, n\}$ and $X$ is the set of "left vertices" and $Y$ is the set of "right vertices" in the complete bipartite graph $K_{X, Y}$. The AP can be stated in terms of the matrix $C$ as follows. Find a permutation $\pi$ of $[n]=\{1,2, \ldots, n\}$ that minimizes $\sum_{i=1}^{n} C(i, \pi(i))$. Let $\mathrm{AP}(C)$ be the optimal value of the instance of the AP specified by $C$.

The Asymmetric Traveling-Salesman Problem (ATSP) is the problem of finding a Hamiltonian circuit of minimum weight in an edge-weighted directed graph. An instance of the ATSP can be specified by an $n \times n$ matrix $C=(C(i, j))$ in which $C(i, j)$ denotes the weight of edge $(i, j)$. The ATSP can be stated in terms of the matrix $C$ as follows: find a cyclic permutation $\pi$ of $[n]$ that minimizes $\sum_{i=1}^{n} C(i, \pi(i))$; here a cyclic permutation is one whose cycle structure consists of a single cycle. Let $\operatorname{ATSP}(C)$ be the optimal value of the instance of the ATSP specified by $C$.

It is evident from the parallelism between the above two definitions that $\operatorname{AP}(C) \leq$ $\operatorname{ATSP}(C)$. The ATSP is NP-hard, whereas the AP is solvable in time $O\left(n^{3}\right)$. Several authors have investigated whether the AP can be used effectively in a branch-and-bound method to solve the ATSP and have observed that the AP gives extremely good bounds on random instances.

Karp was able to explain this in an important paper [11]. He assumed that the entries of $C$ were independent uniform $[0,1]$ random variables, and proved the surprising result that

$$
\mathbf{E}(\operatorname{ATSP}(C)-\operatorname{AP}(C))=o(1)
$$

Since whp $^{1} \mathrm{AP}(C)>1$ we see that this rigorously explains the quality of the assignment bound, a significant plus for probabilistic analysis. Importantly, Karp proved (1) constructively, analysing an $O\left(n^{3}\right)$ patching heuristic that transformed an optimal Assignment Problem solution into a good TSP solution. Karp and Steele [12] simplified and sharpened this

\footnotetext{
${ }^{*}$ Research supported by NSF grant CCR-9818411, Department of Mathematical Sciences, Carnegie Mellon University, Pittsburgh PA15213, e-mail alan@random.math.cmu.edu

${ }^{\dagger}$ Department of Mathematical Sciences, IBM T.J. Watson Research Center, Yorktown Heights NY 10598, e-mail sorkin@watson. ibm.com

${ }^{1}$ with high probability, i.e., with probability 1-o(1) as $n \rightarrow \infty$
} 
analysis, and Dyer and Frieze [4] improved the error bound in (1) to $O\left(\frac{(\ln n)^{4}}{n \ln \ln n}\right)$. Our first theorem sharpens this further.

Theorem 1 Over random cost matrices $C$,

$$
\begin{aligned}
\operatorname{ATSP}(C)-\operatorname{AP}(C) & \leq c_{1} \frac{(\ln n)^{2}}{n} \\
\mathbf{E}(\operatorname{ATSP}(C)-\operatorname{AP}(C)) & \geq \frac{c_{0}}{n}
\end{aligned}
$$

In this paper, $c_{0}, c_{1}, \ldots$ are positive absolute constants whose precise values are not too important to us.

As in previous works, we will prove the upper bound in Theorem 1 by analysing an $O\left(n^{3}\right)$ heuristic which patches an optimal AP solution into a good ATSP solution. We note a related result of Frieze, Karp and Reed [8], who consider the $C(i, j)$ to be random positive integers chosen from the range $[0, L=L(n)]$, and determine when one has ATSP $=\mathrm{AP}$ whp.

Karp and Steele showed that whp the greatest cost of an edge used in the optimal assignment was $O\left(\frac{(\ln n)^{2}}{n}\right)$; our next theorem improves upon this. Let $C_{\max }=C_{\max }(C)$ denote the maximum cost of an edge used in an optimal assignment.

Theorem 2 Whp over random cost matrices $C$,

$$
(1-o(1)) \frac{\ln n}{n} \leq C_{\max } \leq c_{2} \frac{\ln n}{n} .
$$

It is perhaps of interest to estimate the expected difference $\Delta_{2}$ between the cheapest and second-cheapest assignments. (Since 1 in $n$ permutations is cyclic, it is plausible that the ATSP might typically be the $n$ 'th cheapest assignment, providing one reason that gaps between various cheap assignments are a natural object of study.)

Theorem 3 Over random cost matrices $C, \mathbf{E}\left(\Delta_{2}\right) \leq c_{3} \frac{\ln n}{n^{2}}$.

We have no non-trivial lower bound.

The algorithm with the best known worst-case time for solving the ATSP is the $O\left(n^{2} 2^{n}\right)$ dynamic programming algorithm of Held and Karp [9]. The next theorem describes a modest, probabilistic improvement.

Theorem 4 Whp, a random instance of the ATSP can be solved exactly in time $e^{\tilde{\mathrm{O}}(\sqrt{n})}$.

Here $\tilde{O}$ is the standard notation for ignoring logarithmic factors. 


\section{Analysis of the Assignment Problem}

In this section we will prove Theorem 2. The difficult part of the proof - showing that the longest edge in an optimal assignment has length $O(\ln n)$ - has its essence in Lemma 5 below.

Define the $k$-neighborhood of a vertex to be the $k$ vertices nearest it, where distance is given by the matrix $C$; let the $k$-neighborhood of a set be the union of the $k$-neighborhoods of its vertices. In particular, for a complete bipartite graph $K_{X, Y}$ and all $S \subseteq X, T \subseteq Y$,

$$
\begin{aligned}
& N_{k}(S) \doteq\{y \in Y: \exists s \in S \text { s.t. }(s, y) \text { is one of the } k \text { shortest arcs out of } s\} \\
& N_{k}(T) \doteq\{x \in X: \exists t \in T \text { s.t. }(x, t) \text { is one of the } k \text { shortest arcs into } t\} .
\end{aligned}
$$

Given the complete bipartite graph $K_{X, Y}$, any permutation $\pi: X \rightarrow Y$ has an associated matching $M_{\pi}=\{(x, y): x \in X, y \in Y, y=\pi(x)\}$. Given a cost matrix $C$ and permutation $\pi$, define the digraph

$$
\vec{D}=\vec{D}_{C, \pi}=(X \cup Y, \vec{E})
$$

consisting of backwards matching edges and forward "short" edges:

$$
\begin{aligned}
\vec{E}=\{(y, x): y \in Y, x \in X, y=\pi(x)\} \cup\{(x, y): x & \left.\in X, y \in N_{40}(x)\right\} \\
& \cup\left\{(x, y): y \in Y, x \in N_{40}(y)\right\} .
\end{aligned}
$$

Lemma 5 Whp over random cost matrices $C$, for every permutation $\pi$, the diameter of $\vec{D}=\vec{D}_{C, \pi}$ is at most $3 \log _{4} n$.

Proof. For $S \subseteq X, T \subseteq Y$, let

$$
\begin{aligned}
& N_{\vec{D}}(S)=\{y \in Y: \exists s \in S \text { such that }(s, y) \in \vec{E}\} \\
& N_{\vec{D}}(T)=\{x \in X: \exists t \in T \text { such that }(x, t) \in \vec{E}\} .
\end{aligned}
$$

We first prove that whp, for all $S \subseteq X$ with $|S| \leq\lceil n / 5\rceil,\left|N_{\vec{D}}(S)\right| \geq 4|S|$. (Note that only the cheap edges out of $S$, and not the matching edges into it, are involved here.)

$$
\begin{aligned}
\operatorname{Pr}\left(\exists S:|S| \leq\lceil n / 5\rceil,\left|N_{\vec{D}}(S)\right|<4|S|\right) & \leq \sum_{s=1}^{\lceil n / 5\rceil}\left(\begin{array}{c}
n \\
s
\end{array}\right)\left(\begin{array}{c}
n \\
4 s
\end{array}\right)\left(\frac{\left(\begin{array}{c}
4 s \\
40
\end{array}\right)}{\left(\begin{array}{c}
n \\
40
\end{array}\right)}\right)^{s} \\
& \leq \sum_{s=1}^{\lceil n / 5\rceil}\left(\frac{n e}{s}\right)^{s}\left(\frac{n e}{4 s}\right)^{4 s}\left(\frac{4 s}{n}\right)^{40 s} \\
& =\sum_{s=1}^{\lceil n / 5\rceil}\left(\frac{e^{5} 4^{36} s^{35}}{n^{35}}\right)^{s} \\
& =o(1) .
\end{aligned}
$$

Similarly, whp, for all $T \subseteq Y$ with $|T| \leq\lceil n / 5\rceil,\left|N_{\vec{D}}(T)\right| \geq 4|T|$. (Again only the cheap edges, not the matching edges, are involved.) 
In the remainder of this proof, assume that we are in the high-probability "good" case, in which all small sets $S$ and $T$ expand.

Now, for an arbitrary $x \in X$, define $S_{0}, S_{1}, S_{2}, \ldots$, by

$$
S_{0}=\{x\} \text { and } S_{i}=\pi^{-1}\left(N_{\vec{D}}\left(S_{i-1}\right)\right) \text {. }
$$

Since we are in the good case, $\left|S_{i}\right| \geq 4\left|S_{i-1}\right|$ provided $\left|S_{i-1}\right| \leq n / 5$, and so there exists a smallest index $i_{S}-1 \leq \log _{4}(n / 5) \leq \log _{4} n-1$ such that $\left|S_{i_{S}-1}\right|>n / 5$. Arbitrarily discard vertices from $S_{i_{S}-1}$ to create a smaller set $S_{i_{S}-1}^{\prime}$ with $\left|S_{i_{S}-1}^{\prime}\right|=\lceil n / 5\rceil$, so that $S_{i_{S}}^{\prime}=N_{\vec{D}}\left(S_{i_{S}-1}^{\prime}\right)$ has cardinality $\left|S_{i_{S}}^{\prime}\right| \geq 4\left|S_{i_{S}-1}^{\prime}\right| \geq 4 n / 5$.

Similarly, for an arbitrary $y \in Y$, define $T_{0}, T_{1}, \ldots$, by

$$
T_{0}=\{y\} \text { and } T_{i}=\pi\left(N_{\vec{D}}\left(T_{i-1}\right)\right) \text {. }
$$

Again, we will find an index $i_{T} \leq \log _{4} n$ whose modified set has cardinality $\left|T_{i_{T}}^{\prime}\right| \geq 4 n / 5$.

With both $\left|S_{i_{S}}^{\prime}\right|$ and $\left|T_{i_{T}}^{\prime}\right|$ larger than $n / 2$, there must be some $x^{\prime} \in S_{i_{S}}^{\prime}$ for which $y^{\prime}=$ $\pi\left(x^{\prime}\right) \in T_{i_{T}}^{\prime}$. This establishes the existence of a walk and hence a path of length at most $2\left(i_{S}+i_{T}\right) \leq 2 \log _{4} n$ from $x$ to $y$ in $\vec{D}$.

We have proved there is a short path from any $x \in X$ to any $y \in Y$. A short path from $x$ to $x^{\prime}$ both in $X$ can be formed by going from $x$ to $y=\pi\left(x^{\prime}\right)$ and appending the backward edge to $x^{\prime}$; a path from $y$ to $x^{\prime}$ by starting with the backward edge from $y$ to $x=\pi^{-1}(y)$ and then pursuing a path to $x^{\prime}$; and a path from $y$ to $y^{\prime}$ by taking a path from $y$ to $x^{\prime}=\pi^{-1}\left(y^{\prime}\right)$ and discarding its final backward edge.

We will also need the following inequality, Lemma 4.2(b) of [7].

Lemma 6 Suppose that $k_{1}+k_{2}+\cdots+k_{M} \leq a \ln N$, and $Y_{1}, Y_{2}, \ldots, Y_{M}$ are independent random variables with $Y_{i}$ distributed as the $k_{i}$ th minimum of $N$ independent uniform $[0,1]$ random variables. If $\lambda>1$ then

$$
\operatorname{Pr}\left(Y_{1}+\cdots+Y_{M} \geq \frac{\lambda a \ln N}{N+1}\right) \leq N^{a(1+\ln \lambda-\lambda)} .
$$

Let the weight of a forward edge $(x, y)$ be $C(x, y)$ and the weight of backwards edge $(y, x)$ be $-C(x, y)$.

Lemma 7 Whp over random $C$, for all $\pi$, the weighted diameter of $\vec{D}=\vec{D}_{C, \pi}$ is $\leq c_{2} \frac{\ln n}{n}$. Proof. Let

$$
Z_{1}=\max \left\{\sum_{i=0}^{k} C\left(x_{i}, y_{i}\right)-\sum_{i=0}^{k-1} C\left(y_{i}, x_{i+1}\right)\right\},
$$

where the maximum is over sequences $x_{0}, y_{0}, x_{1}, \ldots, x_{k}, y_{k}$ where $\left(x_{i}, y_{i}\right)$ is one of the 40 shortest arcs leaving $x_{i}$ for $i=0,1, \ldots, k \leq k_{0}=\left\lceil 3 \log _{4} n\right\rceil$.

We estimate the probability that $Z_{1}$ is large. Indeed, for any $\zeta>0$ we have

$$
\begin{aligned}
\operatorname{Pr}\left(Z_{1} \geq \zeta \frac{\ln n}{n}\right) \leq \sum_{k=1}^{k_{0}} n^{2 k+1} \frac{1}{(n-1)^{k}} \frac{1}{k !} \times \\
\int_{y=0}^{\infty}\left[\left(\frac{y \ln n}{n}\right)^{k} \sum_{\rho_{1}+\cdots+\rho_{k} \leq 40 k} q\left(\rho_{1}, \ldots, \rho_{k} ; \zeta+y\right)\right] d y
\end{aligned}
$$


where

$$
q\left(\rho_{1}, \ldots, \rho_{k} ; \eta\right)=\operatorname{Pr}\left(X_{1}+\cdots+X_{k} \geq \eta \frac{\ln n}{n}\right),
$$

$X_{1}, \ldots, X_{k}$ are independent and $X_{j}$ is distributed as the $\rho_{j}$ th minimum of $n-1$ uniform $[0,1]$ random variables.

Explanation: We have $\leq n^{2 k+1}$ choices for the sequence $x_{0}, y_{0}, x_{1}, \ldots, x_{k}, y_{k}$. The term $\frac{1}{k !}\left(\frac{y \ln n}{n}\right)^{k}$ bounds the probability that the sum of $k$ independent uniforms, $C\left(y_{0}, x_{1}\right)+\cdots+$ $C\left(y_{k-1}, x_{k}\right)$, is at most $\frac{y \ln n}{n}$. We integrate over $y \cdot \frac{1}{n-1}$ is the probability that $\left(x_{i}, y_{i}\right)$ is the $\rho_{i}$ th shortest edge leaving $x_{i}$, and these events are independent for $0 \leq i \leq k-1$. The final summation bounds the probability that the associated edge lengths sum to at least $\frac{(\zeta+y) \ln n}{n}$.

It follows that if $\zeta$ is sufficiently large then, for all $y \geq 0, q\left(\rho_{1}, \ldots, \rho_{i} ; \zeta+y\right) \leq n^{-\left(\xi^{n}+y\right) / 2}$ and

$$
\begin{aligned}
\operatorname{Pr}\left(Z_{1} \geq \zeta \frac{\ln n}{n}\right) & \leq 2 n^{1-\zeta / 2} \sum_{k=1}^{k_{0}} \frac{(\ln n)^{k}}{k !}\left(\begin{array}{c}
40 k-1 \\
k-1
\end{array}\right) \int_{y=0}^{\infty} y^{k} n^{-y / 2} d y \\
& \leq 2 n^{1-\zeta / 2} \sum_{k=1}^{k_{0}} \frac{(\ln n)^{k}}{k !}\left(\frac{40 e}{\ln n}\right)^{k+1} \Gamma(k+1) \\
& \leq 2 n^{1-\zeta / 2}(40 e)^{k_{0}+2} \\
& =o(1)
\end{aligned}
$$

for large enough $\zeta$. Similarly, whp $Z_{2} \leq \zeta \frac{\ln n}{n}$, where $Z_{2}$ is the maximum of the RHS of expression (7) over sequences where $\left(x_{i}, y_{i}\right)$ is one of the 40 shortest arcs entering $y_{i}$.

An alternating path $P$ from $x \in X$ to $y \in Y$ defined in Lemma 5 can be decomposed into a path $P_{1}$ from $x$ to $y^{\prime}=\pi\left(x^{\prime}\right)$, the edge $\left(y^{\prime}, x^{\prime}\right)$ and a path $P_{2}$ from $x^{\prime}$ to $y$. The cost of $P$ is at most the sum of the costs of $P_{1}, P_{2}$ which is at most $Z_{1}+Z_{2} \leq 2 \zeta \frac{\ln n}{n}$ whp.

We have proved there is a cheap path from any $x \in X$ to any $y \in Y$. Extending this to cheap paths between any two vertices is just as in the proof of Lemma 5 .

We can now prove Theorem 2 , restated here for convenience.

Theorem 2 Whp over random cost matrices $C$,

$$
(1-o(1)) \frac{\ln n}{n} \leq C_{\max } \leq c_{2} \frac{\ln n}{n} .
$$

Proof. The lower bound follows easily from the fact that $\frac{\ln n}{n}$ is the threshold probability for a random bipartite graph to have a perfect matching, as shown by Erdős and Rényi [6].

For the upper bound, define $\vec{D}=\vec{D}_{C, \pi}$ as per (4) and (5). From the preceding lemma, we can assume the existence of a cheap alternating path from any $x$ to $\pi(x)$,

$$
x=x_{0}, y_{0}, x_{1}, y_{1}, \ldots, x_{k}, y_{k}=\pi(x), \quad k \leq 2 k_{0}
$$

consisting of cheap forward edges and backwards matching edges. Appending a final backwards edge $(\pi(x), x)$ creates an alternating cycle.

If any edge in the optimal matching has cost $C(x, \pi(x))>\frac{c_{2} \ln n}{n}$, then the canonical alternating cycle on $x$ has reverse (matching) edge cost at least $\frac{c_{2} \ln n}{n}$ yet whp has forward 
edge cost $Z_{x} \leq \frac{c_{2} \ln n}{n}$. From the original matching, delete the alternating cycle's matching edges and replace them with its forward edges to produce a new matching of smaller cost - contradicting optimality. Thus whp, every edge in an optimal matching has cost $C(x, \pi(x)) \leq \frac{c_{2} \ln n}{n}$.

\section{Analysis of the Traveling Salesman Problem}

Our goal in this section is to prove Theorem 1, recalled here for convenience.

Theorem 1 Over random cost matrices $C$,

$$
\begin{aligned}
\operatorname{ATSP}(C)-\operatorname{AP}(C) & \leq c_{1} \frac{(\ln n)^{2}}{n} \\
\mathbf{E}(\operatorname{ATSP}(C)-\operatorname{AP}(C)) & \geq \frac{c_{0}}{n} .
\end{aligned}
$$

We prove the Theorem's first assertion in sections through 3.3, and the second in section 3.4.

If $(i, \pi(i)), i \in X$, is a perfect matching of $K_{X, Y}$, then $(i, \pi(i))$ defines a permutation digraph, i.e., a set of vertex-disjoint directed cycles that cover all $n$ vertices of the complete directed graph $\vec{K}_{n}$ associated with $K_{X, Y}$. The size $|\pi|$ of $\pi$ is the number of cycles in the permutation.

Similarly a near-perfect matching gives rise to a near-permutation digraph (NPD), i.e., a digraph obtained from a permutation digraph by removing one edge. Thus an NPD $\Gamma$ consists of any number of directed cycles and a single directed path $\operatorname{PATH}(\Gamma)$.

The edges $(i, j)$ will be coloured: Red for $C(i, j) \in\left[0, c_{2} \frac{\ln n}{n}\right] ;$ Blue for $C(i, j) \in\left(c_{2} \frac{\ln n}{n}, 2 c_{2} \frac{\ln n}{n}\right] ;$ Green for $C(i, j) \in\left(2 c_{2} \frac{\ln n}{n}, 3 c_{2} \frac{\ln n}{n}\right] ;$ and Black otherwise.

We will use a three phase method as outlined below:

Phase 1. Solve the assignment problem to obtain an optimal assignment $\pi$ and perfect matching $M_{\pi}$ in $K_{X, Y}$; whp, only Red edges are used.

Phase 2. Whp, at cost $O\left(\frac{(\ln n)^{2}}{n}\right)$ we increase the minimum cycle length in the permutation digraph to at least $n_{0}=\left\lceil\frac{n}{(\ln n)^{5 / 3}}\right\rceil$. We use Red and Blue edges.

Phase 3. Whp, at cost $O\left(\frac{(\ln n)^{2}}{n}\right)$ we convert the Phase 2 permutation digraph to a tour. We use Green edges.

\subsection{Phase 1}

That only Red edges are used in an optimal assignment is immediate from Theorem 2. Also, whp, the optimal assignment $\pi$ 's associated permutation digraph $\Pi_{1}$ is of size $\left|\Pi_{1}\right| \leq 2 \ln n$. This holds because $\pi$ is a random permutation; we will elaborate on this in Phase 2 . 


\subsection{Phase 2}

In this phase, to increase the minimum cycle length in the $\mathrm{PD}$, we will deal with each small cycle in turn. Let us describe the essence of how one small cycle of a PD is repaired, setting aside the combinatorial and probabilistic issues. One edge $(a, b)$ of the cycle is chosen. From vertex $a$, an alternating path is grown, alternating forward non-PD edges (starting with an edge out of $a$ ) with PD edges traversed backwards. From $b$ a similar path is grown, alternating non-PD edges traversed backwards (starting with an edge into $b$ ) with PD edges traversed forwards. The $a$-path, followed by the edge joining its terminal to that of the $b$-path, followed by the reversed $b$-path, followed by the edge $(b, a)$, defines an alternating cycle. The "sum" of this cycle and the original PD is a new PD. If the two paths, and the edge bridging their endpoints, are cheap, the new PD is not much more expensive than the old one. How does the new structure compare with the old one?

Consider the sum of the original PD and the path on $a$, as the path grows. When the path enters a vertex on a PD cycle and exits from the vertex's predecessor, the sum (an NPD) includes a directed path starting at $a$ and going the long way around through the cycle. When the next cycle is struck, it is added to this string. If a cycle is hit a second time ("the string crosses itself"), the loop formed splits off as a cycle, and the path continues on. Similarly from $b$. As long as no cycles split off are small, and $a$ and $b$ hit no common cycles, and either $a$ or $b$ hits at least one large cycle, the new cycle containing $a$ and $b$, and any other new cycles formed, will be large. We will try to arrange for this to be the case, otherwise declaring the attempt a failure.

If we fail for one edge $(a, b)$ in a cycle, we try for one more (unless the cycle size is 10 or less). Since we will never make any new small cycles, the "trial" edges can all be fixed in advance, randomly. If we fail for a cycle, then the entire algorithm fails. If we succeed, we proceed to the next small cycle, until all small cycles are repaired.

Of course the "new" PD of one case becomes the "original" PD of the next one, and the most difficult part of the analysis is avoiding conditioning that might be introduced by this evolving cycle structure. (We will rely on the fact that a PD is induced by a bipartite matching when the two sets of vertices are put into correspondence by a labelling, and until that labelling is established, the PD and the matching are in a sense independent.)

The first detail is the construction of the cheap alternating paths out of vertices $a$ and $b$. Paths alternating with respect to a PD as described above are also alternating with respect to the corresponding bipartite matching. We begin by finding a cheap "alternating tree" (actually a directed acyclic graph, or DAG, but no matter), rooted at $a$, containing many cheap alternating paths. After doing the same for $b$, we (hopefully) find some cheap edge between an $a$-leaf and a $b$-leaf, and we use the paths selected by these leaves.

To define the trees, recall the definitions (2) and (3) of $N_{k}(S)$ and $N_{k}(T)$. For the remainder of this section let $K$ be a suitably large constant. Let $E_{K}=\{(x, y): y \in$ $N_{K}(x)$ or $\left.x \in N_{K}(y)\right\}$.

Lemma $8 \mathrm{Whp}$ over random matrices $C$, every set of $s \leq s_{0}=\frac{\ln n}{2 \ln \ln n}$ vertices, spans at most $s$ edges from $E_{K}$.

Proof. Since $K$ is large, we know that whp every edge in $E_{K}$ has length at most $2 K \frac{\ln n}{n}$. 
So the probability there exists a set $S$ containing $|S|+1$ edges is at most

$$
o(1)+\sum_{s=1}^{s_{0}}\left(\begin{array}{l}
n \\
s
\end{array}\right)\left(\begin{array}{c}
s(s-1) \\
s+1
\end{array}\right)\left(2 K \frac{\ln n}{n}\right)^{s+1} \leq o(1)+\sum_{s=1}^{s_{0}} \frac{s}{n}\left(2 e^{2} K \ln n\right)^{s+1}=o(1)
$$

Lemma 9 Whp over random matrices $C$, for all $S \subseteq X, T \subseteq Y$, with $|S|,|T| \leq n^{3 / 4}$,

$$
\left|N_{K}(S)\right| \geq(K-2)|S| \text { and } N_{K}(T)|\geq(K-2)| T \mid
$$

Proof. Just as in deriving (6),

$$
\begin{aligned}
& \operatorname{Pr}(\exists S \text { or } T: \neg(9)) \\
& \leq 2 \sum_{s=1}^{n^{3 / 4}}\left(\begin{array}{l}
n \\
s
\end{array}\right)\left(\begin{array}{c}
n \\
(K-2) s
\end{array}\right)\left(\frac{\left(\begin{array}{c}
(K-2) s \\
K
\end{array}\right)}{\left(\begin{array}{l}
n \\
K
\end{array}\right)}\right)^{s} \\
& =o(1) .
\end{aligned}
$$

As before, we use this expansion to create many short alternating paths. Let a bijection (matching) $\rho_{i}$ between $X$ and $Y$ be given, and let one matching edge $\left(a_{i}, b_{i}\right)$ be specified. Define branching factors $r_{1}=\lceil K \ln n\rceil$ for a first generation $t=1$, and $r_{t}=K$ for all subsequent generations $t \geq 2$. For each $i$ we construct a "tree" (actually a DAG) which we will use to modify bijection $\rho=\rho_{i}$. Its depth- $t$ nodes consist of the set $S_{i}^{(t)}\left(\operatorname{resp}\right.$. $\left.T_{i}^{(t)}\right)$. The depth-0 node set is the singleton consisting of the given vertex itself,

$$
S_{i}^{(0)}=\left\{a_{i}\right\} \text { and } T_{i}^{(0)}=\left\{b_{i}\right\}
$$

For $1 \leq t \leq \frac{\ln n}{2 \ln \ln n}$ let

$$
S_{i}^{(t)}=\rho^{-1}\left(N_{r_{t}}\left(S_{i}^{(t-1)}\right)\right) \text { and } T_{i}^{(t)}=\rho\left(N_{r_{t}}\left(T_{i}^{(t-1)}\right)\right)
$$

and for $t>\frac{\ln n}{2 \ln \ln n} \operatorname{let}$

$$
\begin{aligned}
& S_{i}^{(t)}=\rho^{-1}\left(N_{r_{t}}\left(S_{i}^{(t-1)}\right)\right) \backslash\left(\bigcup_{i^{\prime}<i} S_{i^{\prime}}^{(1)} \cup \bigcup_{i^{\prime}<i} \rho^{-1}\left(T_{i^{\prime}}^{(1)}\right)\right) \\
& T_{i}^{(t)}=\rho\left(N_{r_{t}}\left(T_{i}^{(t-1)}\right)\right) \backslash\left(\bigcup_{i^{\prime}<i} T_{i^{\prime}}^{(1)} \cup \bigcup_{i^{\prime}<i} \rho\left(S_{i^{\prime}}^{(1)}\right)\right) .
\end{aligned}
$$

It follows from Lemmas 8 and 9 that whp $\left|S_{i}^{(t)}\right| \geq(K-3)\left|S_{i}^{(t-1)}\right|$ and $\left|T_{i}^{(t)}\right| \geq(K-3)\left|T_{i}^{(t-1)}\right|$ as long as both $S_{i}^{(t-1)}$ and $T_{i}^{(t-1)}$ are of size at most $n^{3 / 4}$. By throwing away random vertices if necessary, we can assume that $\left|S_{i}^{(t)}\right|=(K-3)\left|S_{i}^{(t-1)}\right|$ and $\left|T_{i}^{(t)}\right|=(K-3)\left|T_{i}^{(t-1)}\right|$. Thus if $\tau=\left\lceil 1+\log _{K-3}\left(n^{3 / 4} /(K \ln n)\right)\right\rceil$, then whp

$$
\forall i: n^{3 / 4} \leq\left|S_{i}^{(\tau)}\right|=\left|T_{i}^{(\tau)}\right| \leq K n^{3 / 4}
$$


Each $x \in S_{i}^{(t)}$ defines a walk from $a_{i}$ to $x$, of length $2 t$, which is alternating w.r.t. the matching $M_{\rho} ;$ prune it to define a path $P[i, x]$. Similarly, each $y \in T_{i}^{(t)}$ defines a path $Q[i, y]$ from $y$ to $b_{i}$, of length $2 t$, which is alternating w.r.t. $M_{\rho}$.

We say that a cycle $C$ of $\Pi_{1}$ is small if $|C|<n_{0} . \quad\left(\right.$ Recall that $\left.n_{0}=\left[\frac{n}{(\ln n)^{5 / 3}}\right].\right)$ Let the small cycles of $\Pi_{1}$ be $C_{1}, C_{2}, \ldots$ At the start of Phase 2, from each cycle $C$ of length less than 10 we choose an edge $(a, b)$ of $C, a \in X, b \in Y$, and for each small cycle of length 10 or more, we choose a second one disjoint from the first. Let the chosen edges be $\left(a_{i}, b_{i}\right), i=1,2, \ldots, \lambda$.

We now describe how we try to remove a $C_{j}$ without creating any new small cycles. Suppose we have removed $C_{1}, C_{2}, \ldots, C_{j-1}$ and the original permutation $\pi$ has become $\rho=$ $\rho_{i}$. Assume that we have not already serendipitously removed $C_{j}$ as well. Let $\left(a_{i}, b_{i}\right)$ be one of the chosen edges of $C_{j}$.

Each alternating path $P[i, x]$ starts with a "forward" edge which is one of the $K \ln n$ shortest edges leaving $a_{i}$ (the first branching factor was $r_{1}=K \ln n$ ), $\tau-1$ other forward edges each of which is one of the $K$ shortest edges leaving a vertex, and another $\tau$ "backward", matching edges (edges in $M_{\rho}$ ); a symmetric condition holds for $Q_{i}$.

It follows from the proof of Lemma 7 that whp each of these paths is such that the total length of its forward edges minus the total length of its backward edges is bounded by $c_{4} \frac{\ln n}{n}$.

We now see that if we find $\xi_{i} \in S_{i}^{(\tau)}$ and $\eta_{i} \in T_{i}^{(\tau)}$ such that $\left(\xi_{i}, \eta_{i}\right)$ is Blue then it together with the edge $\left(a_{i}, b_{i}\right)$ and the paths $P\left[i, \xi_{i}\right]$ and $Q\left[i, \eta_{i}\right]$ - defines an alternating cycle whose action on the current perfect matching increases its cost by at most $\left(c_{4}+2 c_{2}\right) \frac{\ln n}{n}$. We now show that we can whp find at least one such alternating cycle whose action does not create any new small cycles. Furthermore, if such a path contains an edge of $C_{j^{\prime}}, j^{\prime}>j$, then this alternating cycle will also destroy the cycle $C_{j^{\prime}}$.

Let $\phi$ be a random permutation of $[n]$ associating the vertices of $X$ to those of $Y$, and let matrix $\hat{C}$ be defined by $\hat{C}(i, j)=C(i, \phi(j))$. If $\psi$ is the (w.p. 1 , unique) minimum solution to the assignment problem with matrix $\hat{C}$ then $\pi=\phi \psi$ is the minimum solution to the original problem. We exploit the randomness of $\phi$ which produces the random permutation $\pi$ from $\psi$. Instead of taking $\pi$ as given, we assume that $\psi$ is given and $\pi$ is to be obtained through a random permutation $\phi$. We condition on the cycle structure of $\pi$. We assume that there are $k_{i}$ cycles of length $i$ and that (i) $\sum_{i=1}^{n_{0}} i k_{i} \leq 2 n_{0}$ and that (ii) $\sigma=\sum_{i=1}^{n} k_{i} \leq 2 \ln n$. These conditions hold whp. How do we sample from this conditional distribution? Let $\Pi$ denote the set of permutations of $X$ with the given cycle structure. For $j \in[\sigma]$ let $C_{j}=\left\{t_{j}+1, \ldots, t_{j+1}\right\}$ where $t_{1}=0, t_{\sigma+1}=n$, and the multi-sets $\left\{t_{j+1}-t_{j}: j \in[\sigma]\right\}$ and $\left\{k_{i} \times i: i \in[n]\right\}$ coincide. We first define $\gamma:$ if $x, y \in C_{j}$ and $y=x+1 \bmod t_{j+1}-t_{j}$ then $\gamma(x)=y$. Then given a bijection $f: X \rightarrow[n]$ we define a permutation $\pi_{f}$ on $X$ by $\pi_{f}=f^{-1} \gamma f$. Each permutation $\pi \in \Pi$ appears precisely $\prod_{i=1}^{n} k_{i} ! i^{k_{i}}$ times as $\pi_{f}$. Thus choosing a random mapping $f$, chooses a random $\pi_{f}$ from $\Pi$. (This is equivalent to randomly choosing $\phi=\psi^{-1} f^{-1} \gamma f$.) The most natural way to look at this is to think of having oriented cycles on the plane whose vertices are at points $P_{1}, P_{2}, \ldots, P_{n}$ and then randomly labelling these points with 1 to $n$. Then if $P^{\prime}$ follows $P$ on one of the cycles and $P, P^{\prime}$ are labelled $x, x^{\prime}$ by $f$ then $\pi_{f}(x)=x^{\prime}$.

As we proceed through Phase 2 we have to expose parts of $f$ (equivalently $\phi$ ). $x$ is clean if $f(x)$ is unexposed (the label $x$ has not yet been used) and dirty otherwise. Thus imagine 
that we have cycles, mostly unlabelled, but with a few vertices labelled. Let us use to denote a partially labelled graph.

We can now describe how to eliminate the small cycles. We proceed in order $i \in[\lambda]$. At stage $i$ we should have eliminated $C_{1}, C_{2}, \ldots, C_{j-1}$ for some $j$ and have a current perfect matching $M_{i}$, defining $\rho_{i}$. (Consider $M_{i}$ to be fully revealed, but the labels on its vertices not revealed except for those matching edges in short cycles; thus all that is revealed of $\rho_{i}$ is its cycle structure and labels on these few edges.)

We construct the trees $S_{i}^{(\tau)}$ and $T_{i}^{(\tau)}$, and then seek a blue edge between the leaves of $S_{i}^{(\tau)}$ and those of $T_{i}^{(\tau)}$. The unconditional probability there are fewer than $\frac{1}{2} n^{1 / 2} \ln n$ such edges is $1-e^{-\Omega\left(n^{1 / 2} \ln n\right)}$ and this bound holds conditionally on there being at least this number for each $i^{\prime}<i$, by the FKG inequality. Choose one $\xi_{i} \in S_{i}^{(\tau)}, \eta_{i} \in T_{i}^{(\tau)}$ such that $\left(\xi_{i}, \eta_{i}\right)$ is Blue. (We will say more later about how to make this choice.) Consider the alternating cycle $C=P\left[i, \xi_{i}\right],\left(\xi_{i}, \eta_{i}\right)$, reversal $Q\left[i, \eta_{i}\right],\left(b_{i}, a_{i}\right)$ (here we may remove some edges to make a cycle that contains $\left(b_{i}, a_{i}\right)$ ). We will define what it means for a cycle to be "acceptable", and show that $C$ is likely to be.

For any $x \in S_{i}^{(t)}$, consider $P[i, x]=\left(x_{0}=a_{i}, y_{0}, x_{1}, y_{1}, \ldots, x_{t}=x\right)$ where $y_{j-1}=\rho_{i}\left(x_{j}\right)$ for $j \geq 1$. $P[i, x]$ defines a sequence $M^{(0)}, M^{(1)}, \ldots, M^{(t)}$ of near-perfect matchings where $M^{(0)}=M_{i} \backslash\left\{\left(a_{i}, b_{i}\right)\right\}$ and $M^{(s)}=\left(M^{(s-1)} \backslash\left\{\left(x_{s}, y_{s-1}\right)\right\}\right) \cup\left\{\left(x_{s}, y_{s}\right)\right\}$. Let $\Gamma^{(0)}, \Gamma^{(1)}, \ldots, \Gamma^{(t)}$ be the associated NPD's. We say that $\Gamma^{(s)}$ is acceptable if (i) $\left|\operatorname{PATH}\left(\Gamma^{(s)}\right)\right| \geq n_{0}$ and (ii) the small cycles of $\Gamma^{(s)}$ are a subset of $C_{i+1}, \ldots, C_{\lambda}$. We say that $x$ is acceptable if $\Gamma^{(0)}, \Gamma^{(1)}, \ldots, \Gamma^{(t)}$ are all acceptable.

Going back to $P\left[i, x=x_{t}\right]$ let us estimate the probability that $x_{t}$ is acceptable, given that it is clean and $x_{t-1}$ is acceptable. Assume that we have chosen $f\left(x_{t-1}\right)$ and that we have a partially labelled NPD $\tilde{\Gamma}_{i}^{(t-1)}$. We randomly choose $f\left(x_{t}\right)$ from the unlabelled points and label it with $x_{t}$. We then replace the $\operatorname{arc}\left(f\left(x_{t}\right), \cdot\right)$ of $\tilde{\Gamma}_{i}^{(t-1)}$ by $\left(f\left(x_{t-1}\right), \cdot\right)$. $x_{t}$ will be acceptable if $f\left(x_{t}\right)$ is not within $n_{0}$ of an endpoint of $\operatorname{PATH}\left(\tilde{\Gamma}_{i}^{(t-1)}\right)$. Since at most $O\left((\ln n)^{2}\right)$ values of $f$ are exposed, this happens with probability at least $1-\frac{2 n_{0}}{n-o(n)}=1-O\left((\ln n)^{-5 / 3}\right)$ conditional on previous exposures. A similar analysis holds for the paths $Q[i, y]$.

If all vertices on $C$ are clean then the probability that $C$ is not acceptable is $O\left((\ln n)^{-2 / 3}\right)$. So the probability that for small cycles of length 10 or more, both trials fail, is $O\left((\ln n)^{-4 / 3}\right)$. Thus if we can always find clean paths then the probability that Phase 2 fails is $o(1)+$ $O\left((\ln \ln n)(\ln n)^{-2 / 3}+(\ln n)(\ln n)^{-4 / 3}\right)=o(1)$, using $\ln \ln n$ as a high probability bound on the number of cycles of length at most 10 in a random permutation. To ensure that we only have to deal with clean cycles, we choose $\xi_{i}, \eta_{i}$ so that $P\left[i, \xi_{i}\right]$ and $Q\left[i, \eta_{i}\right]$ are clean. Can this be done?

Let $A_{i}^{(t)}$ denote the clean vertices of $S_{i}^{(t)}, t=1,2, \ldots, \tau$. It follows from Lemma 8 that $\left|A_{i}^{(1)}\right| \geq K \ln n-4 \tau$ and in general, for $t \geq 1$, from Lemma 9 that $\left|A_{i}^{(t+1)}\right| \geq(K-3)\left|A_{i}^{(t)}\right|-$ $4 \tau \ln n$. Thus, $\left|A_{i}^{(\tau)}\right| \geq n^{3 / 5}$. Making a similar argument for clean vertices of $T_{i}^{(\tau)}$, there is almost sure to be a Blue edge between a clean vertex in $S_{i}^{(\tau)}$ and one in $T_{i}^{(\tau)}$, and they define a clean cycle. 


\subsection{Phase 3}

For Phase 3 we use the Green edges. Now we can easily show that whp a random permutation has a cycle $C$ whose length is in the range $\left[n_{1}=n(\log n)^{-1 / 6}, n(\log n)^{-1 / 7}\right]$. Prior to Phase 2, we fix such a $C$ in our unlabelled set of cycles and expose $f^{-1}(C)$. This defines a random set of matching edges of $M_{\pi}$ whose vertices will be taken out of the Phase 2 process. This will not affect the previous analysis (more details in final version) and we assert that whp at the end of Phase 2, all cycles are of length at least $n_{0}$ and there is at least one cycle of length at least $n_{1}$. Given two cycles $C_{1}, C_{2}$ with one of length at least $n_{0}$ and the other of length at least $n_{1}$, the probability that we cannot patch them together (delete edges $\left(a_{i}, b_{i}\right)$ from $C_{i}, i=1,2$ and replace them by Green edges $\left.\left(a_{1}, b_{2}\right),\left(a_{2}, b_{1}\right)\right)$ is $\left(1-\frac{c_{2}^{2}(\ln n)^{2}}{n^{2}}\right)^{n_{0} n_{1}} \leq e^{-c_{2}^{2}(\ln n)^{1 / 6}}$. Doing this $\leq 2 \ln n$ times increases the cost by at most $4 c_{2} \frac{(\ln n)^{2}}{n}$ and so Phase 3 succeeds whp.

This completes the proof of the high probability upper bound on $A T S P-A P$. We now consider the lower bound.

\subsection{Proof of the lower bound}

The Assignment Problem can be expressed as a linear program:

$$
\text { Minimise } \sum_{i, j} C(i, j) z_{i, j} \text { subject to } \sum_{i} z_{i, k}=\sum_{j} z_{k, j}=1, \forall k, 0 \leq z_{i, j} \leq 1, \forall i, j \text {. }
$$

This has the dual linear program:

$$
\text { Maximise } \sum_{i} u_{i}+\sum_{j} v_{j} \text { subject to } u_{i}+v_{j} \leq C(i, j), \forall i, j \text {. }
$$

Remark 10 Condition on an optimal basis for (LP). We may w.l.o.g. take $u_{1}=0$ in (DLP), whereupon w.p. 1 the other dual variables are uniquely determined. Furthermore, the reduced costs of the non-basic variables $\bar{C}(i, j)=C(i, j)-u_{i}-v_{j}$ are independently and uniformly distributed, with $\bar{C}(i, j) \in_{\text {unif }}\left[\max \left\{0,-u_{i}-v_{j}\right\}, 1-u_{i}-v_{j}\right]$.

Proof. The $2 n-1$ dual variables are unique w.p. 1 because they satisfy $2 n-1$ linear equations. The only conditions on the non-basic edge costs are that $C(i, j) \in[0,1]$ (equivalently $\left.\bar{C}(i, j) \in\left[-u_{i}-v_{j}, 1-u_{i}-v_{j}\right]\right)$ and $\bar{C}(i, j) \geq 0$; intersecting these intervals yields the last claim.

\section{Lemma 11 Whp}

$$
\max _{i, j}\left\{\left|u_{i}\right|,\left|v_{j}\right|\right\} \leq c_{5} \frac{\ln n}{n}
$$

Proof. Optimal dual values $u_{i}, v_{j}$ can be characterised as shortest distances, as follows [1]. Consider a directed bipartite digraph $\Gamma$ on $X \cup Y$ with "forward" edges $\left(x_{i}, y_{j}\right), i, j \in[n]$, $j \neq \pi(i)$, of length $C(i, j)$; and "backward" edges $\left(y_{j}, x_{i}\right), i, j \in[n], j=\pi(i)$, of length $-C(i, \pi(i))$. If $u_{1}=0$, then $-u_{i}$ is the shortest distance $d\left(x_{1}, x_{i}\right)$ from $x_{1}$ to $x_{i}$ in $\Gamma$, and $v_{j}$ is the shortest distance from $x_{1}$ to $y_{j}$. 
Lemma 7 implies that $-u_{i}, v_{i} \leq c_{6} \frac{\ln n}{n}$ for $i \in[n]$. Furthermore, using the fact that a cheapest path is also a cheapest walk (derived from the optimal assignment, $\Gamma$ has no negative-cost cycles $),-u_{j}=d\left(x_{1}, x_{j}\right) \leq d\left(x_{1}, x_{i}\right)+d\left(x_{i}, x_{j}\right) \leq-u_{i}+c_{6} \frac{\ln n}{n}$ implies $u_{i}-$ $u_{j} \leq c_{6} \frac{\ln n}{n}$. Immediately, $\left|u_{i}\right| \leq c_{6} \frac{\ln n}{n}$ and also, with $\bar{u}=\sum \overline{u_{i}} / n,|\bar{u}| \leq c_{6} \frac{\ln n}{n}$. Likewise, $v_{i}-v_{j} \leq c_{6} \frac{\ln n}{n}$, from which $\left|v_{i}-\bar{v}\right| \leq c_{6} \frac{\ln n}{n}$. But we know that whp the optimal assignment cost satisfies $1.51<\sum_{i} u_{i}+\sum_{j} v_{j}<1.94[10,5,13,3]$, so $\bar{v} \in(1.51 / n-\bar{u}, 1.94 / n-\bar{u})$, giving $|\bar{v}| \leq c_{6} \frac{\ln n}{n}+O(1 / n)$ and finally $\left|v_{i}\right| \leq c_{7} \frac{\ln n}{n}$.

Having solved LP we will have $n$ basic variables $z_{i, j},(i, j) \in I_{1}$, with value 1 and $n-1$ basic variables $z_{i, j},(i, j) \in I_{2}$, with value 0 . The edges $\left(x_{i}, y_{j}\right),(i, j) \in I=I_{1} \cup I_{2}$ form a tree $T^{*}$ in $K_{X, Y}$. We show that with probability at least $c_{9}>0$ there exists $(i, i) \in I_{1}$ (a loop) such that $\left(x_{i}, y_{i}\right)$ is a pendant edge in $T^{*}$; w.l.o.g. suppose $x_{i}$ is its leaf. In this case the optimal TSP tour, viewed as a bipartite matching, cannot use the edge $\left(x_{i}, y_{i}\right)$ (a loop), and must use some other edge $\left(x_{i}, y_{i^{\prime}}\right)$; since $x_{i}$ is a leaf in $T^{*}, z_{i, i^{\prime}}$ is not a basic LP variable. The expected value of the reduced cost of $z_{i, i^{\prime}}$ is at least $\frac{c_{10}}{n}$ and so $\mathbf{E}(A T S P-A P) \geq \frac{c_{9} c_{10}}{n}$ and the lower bound follows.

To prove the existence of $(i, i)$ we show that whp the optimal assignment $\psi$ for $\hat{C}$ of Section 3 has at least $c_{11} n$ leaves $L$. After applying the random permutation $\phi$, the number of leaves giving rise to loops is, at least, a random variable whose distribution is asymptotically Poisson with density $c_{11}$; thus

$$
\operatorname{Pr}(\exists \text { at least one leaf-loop }) \geq(1-o(1))\left(1-e^{-c_{11}}\right) .
$$

By taking a spanning tree $T$ of $K_{X, Y}$ which contains a perfect matching $M$ and shrinking the edges of $M$ we obtain a tree isomorphic to a spanning tree $T^{\prime}$ of $K_{n}$. Each $T$ arises from exactly $2^{n-1} T^{\prime}$ s because we have two choices as to how to configure each non- $M$ edge. (An $(i, j)$ edge in $T^{\prime}$ can in $T$ be expanded to $\left(x_{i}, y_{j}\right)$ or to $\left(x_{j}, y_{i}\right)$.) Let $b(T)=b\left(T^{\prime}\right)$ denote the number of branching nodes (degree $\geq 3$ ) of $T$ and $T^{\prime}$. A tree $T^{\prime}$ is $\epsilon$-bushy if $b\left(T^{\prime}\right) \leq \epsilon n$. Bohman and Frieze used this concept in [2] and showed that the number of $\epsilon$-bushy trees is at most $n ! e^{\theta(\epsilon) n}$ where $\theta(\epsilon) \rightarrow 0$ as $\epsilon \rightarrow 0$. It follows that the number of $\epsilon$-bushy trees of $K_{X, Y}$ which have a perfect matching is at most $e^{\theta(\epsilon) n} 2^{n-1} n$ !. Observe that the number of leaves in $T$ is at least $b(T)$. We complete the proof by showing that, for a sufficiently small constant $\epsilon$,

$$
\operatorname{Pr}\left(T^{*} \text { is } \epsilon \text {-bushy }\right)=o(1)
$$

For any tree $T$ with a perfect matching, we can put $u_{1}=0$ and then solve the equations $u_{i}+v_{j}=C(i, j)$ for $\left(x_{i}, y_{j}\right) \in T$ to obtain the associated dual variables. $T$ is optimal if $\bar{C}(i, j)=C(i, j)-u_{i}-v_{j} \geq 0$ for all $\left(x_{i}, y_{j}\right) \notin T$. Let $Z_{T}=\sum_{i} u_{i}+\sum_{j} v_{j}$. Let Now whp the optimal tree $T^{*}$ satisfies $Z_{T^{*}} \in[1.51,1.94]$, because $Z_{T^{*}}$ is the optimal assignment cost, and it is known both that expectation is in the stated range $[13,3]$ and that the actual value is concentrated about the expectation [14]. Then if $\mathcal{E}$ denotes the event $\left\{(11)\right.$ and $Z_{T} \in$ 
$[1.51,1.94]\}$, for any tree $T$, over random matrices $C(i, j)$,

$$
\begin{aligned}
& \operatorname{Pr}\left(Z_{T} \in[1.51,1.94] \text { and }(11) \text { and } \bar{C}(i, j) \geq 0 \forall(i, j) \notin I\right) \\
& \quad \leq \operatorname{Pr}(\bar{C}(i, j) \geq 0 \forall(i, j) \notin T \mid \mathcal{E}) \times \operatorname{Pr}\left(Z_{T} \in[1.51,1.94]\right) \\
& \quad \leq \frac{1.94^{n}}{n !} \mathbf{E}\left(\prod_{\left(x_{i}, y_{j}\right) \notin T}\left(1-\left(u_{i}+v_{j}\right)^{+}\right) \mid \mathcal{E}\right) \\
& \quad \leq \mathbf{E}\left(\exp \left\{-\sum_{\left(x_{i}, y_{j}\right) \notin T}\left(u_{i}+v_{j}\right)\right\} \mid \mathcal{E}\right) \frac{1.94^{n}}{n !} \\
& \left.\quad \leq \mathbf{E}\left(e^{-n Z_{T}} \exp \left\{\sum_{\left(x_{i}, y_{j}\right) \in T}\left(u_{i}+v_{j}\right)\right\} \mid \mathcal{E}\right)\right) \frac{1.94^{n}}{n !} \\
& \quad \leq e^{-1.51 n} n^{2 c_{5}} \frac{1.94^{n}}{n !} .
\end{aligned}
$$

Explanation $\frac{1.94^{n}}{n !}$ bounds the probability that the sum of the lengths of the edges in the perfect matching of $T$ is at most 1.94 . The product term is the probability that each nonbasic reduced cost is non-negative.

Thus

$$
\begin{aligned}
\operatorname{Pr} & \left.\exists \text { an } \epsilon \text {-bushy tree } T: Z_{T} \in[1.51,1.94] \text { and }(11) \text { and } \bar{C}(i, j) \geq 0 \forall(i, j) \notin I\right) \\
& \leq n ! 2^{n} e^{\theta(\epsilon) n} \times e^{-1.51 n} n^{2 c_{5}} \frac{1.94^{n}}{n !} \\
& =o(1)
\end{aligned}
$$

for $\epsilon$ sufficiently small. This implies (12).

\section{An enumerative algorithm}

We can now prove Theorem 4 , restated here for convenience.

Theorem 4 Whp, a random instance of the ATSP can be solved exactly in time $e^{\tilde{\mathrm{O}}(\sqrt{n})}$.

Proof. Let $I_{k}$ denote the interval $\left[2^{-k} c_{1} \frac{(\ln n)^{2}}{n}, 2^{-(k-1)} c_{1} \frac{(\ln n)^{2}}{n}\right]$ for $k \geq 1$. It follows from Lemmas 11 and 10 that whp (i) there are $\leq c_{1} 2^{-(k-1)} n \ln n$ non-basic variables $z_{i, j}$ whose reduced cost is in $I_{k}, 1 \leq k \leq k_{0}=\frac{1}{2} \log _{2} n$ and (ii) there are $\leq 2 c_{1} \sqrt{n} \ln n$ non-basic variables $z_{i, j}$ whose reduced cost is $\leq c_{1} \frac{(\ln n)^{2}}{n^{3 / 2}}$.

We can search for an optimal solution to ATSP by choosing a set of non-basic variables, setting them to 1 and then re-solving the assignment problem. If we try all sets and choose the best tour we find, then we will clearly solve the problem exactly. However, it follows from Theorem 1 that we need only consider sets which contain $\leq 2^{k}$ variables with reduced costs in $I_{k}$ and none with reduced cost $\geq c_{1} \frac{(\ln n)^{2}}{n}$. Thus whp we need only check at most

$$
2^{2 c_{1} \sqrt{n} \ln n} \prod_{k=1}^{k_{0}} \sum_{t=1}^{2^{k}}\left(\begin{array}{c}
c_{1} 2^{-(k-1)} n \ln n \\
t
\end{array}\right)=e^{\tilde{\mathrm{O}}(\sqrt{n})}
$$

sets. 


\section{$5 \quad$ Second best assignment}

We recall and prove Theorem 3 , on the gap $\Delta_{2}$ between the costs of the cheapest and second-cheapest assignments.

Theorem 3 Over random cost matrices $C, \mathbf{E}\left(\Delta_{2}\right) \leq c_{3} \frac{\ln n}{n^{2}}$.

Proof. $\Delta_{2}$ is equal to the minimum non-basic reduced cost. From Lemma 11 and $\sum_{i} u_{i}+$ $\sum_{j} v_{j}>1.51 \mathrm{whp}$, it follows that whp there are at least $n_{1}=c_{7} \frac{n^{2}}{\ln n}$ pairs $i, j$ such that $u_{i}+v_{j}>0$. Each such pair corresponds to a non-basic variable $C_{i j}$, and it follows from Remark 10 that the minimum reduced cost among this set is at most $\frac{1}{n_{1}+1}$ in expectation, proving the theorem.

\section{References}

[1] R.K. Ahuja, T.L. Magnanti and J.B. Orlin, Network Flows Theory, Algorithms and Applications, Prentice-Hall, 1993.

[2] T.Bohman and A.M.Frieze, Avoiding a giant component, to appear.

[3] D. Coppersmith and G.B. Sorkin, Constructive bounds and exact expectations for the random assignment problem, Random Structures and Algorithms 15 (1999), 133-144.

[4] M.E. Dyer and A.M. Frieze, On patching algorithms for random asymmetric traveling salesman problems, Mathematical Programming 46 (1990) 361-378.

[5] M.E. Dyer, A.M. Frieze and C. McDiarmid, Linear programs with random costs, Mathematical Programming 35 (1986) 3-16.

[6] P. Erdős and A. Rényi, On random matrices, Publ. Math. Inst. Hungar. Acad. Sci. 8 (1964) 455-461.

[7] A.M. Frieze and G.R. Grimmett, The shortest path problem for graphs with random arc-lengths, Discrete Applied Mathematics 10 (1985) 57-77.

[8] A.M. Frieze, R.M. Karp and B. Reed, When is the assignment bound asymptotically tight for the asymmetric traveling-salesman problem?, SIAM Journal on Computing 24 (1995) 484-493.

[9] M. Held and R.M. Karp, A dynamic programming approach to sequencing problems, SIAM Journal of Applied Mathematics 10 (1962) 196-210.

[10] R.M. Karp, An upper bound on the expected cost of an optimal assignment, Discrete Algorithms and Complexity: Proceedings of the Japan-US Joint Seminar (D. Johnson et al., eds.) 1-4, Academic Press, New York, 1987.

[11] R.M. Karp, A patching algorithm for the non-symmetric traveling salesman problem, SIAM Journal on Computing 8 (1979) 561-573. 
[12] R.M. Karp and J.M. Steele, Probabilistic analysis of heuristics, in The traveling salesman problem: a guided tour of combinatorial optimization, E.L. Lawler, J.K. Lenstra, A.H.G. Rinnooy Kan and D.B. Shmoys Eds. (1985) 181-206.

[13] B. Olin, Asymptotic properties of random assignment problems, Ph.D. thesis, Kungl Tekniska Högskolan, Stockholm, Sweden, 1992.

[14] D. Aldous, Asymptotics in the random assignment problem, Probability Theory and Related Fields 93 (1992) 507-534.

This research was sponsored in part by National Science Foundation (NSF) grant no. CCR-0122581. 